\title{
LETTERS
}

doi:10.1017/S1041610209991190

\section{Predicting memory decline as a risk factor for Alzheimer's disease in older post-menopausal women: quod erat demonstrandum?}

Alzheimer's disease (AD) is the major form of agerelated dementia worldwide, accounting for more than two-thirds of all dementia cases. The disease is characterized by a progressive loss of cognitive and intellectual functioning (Gilman, 1997). A number of risk factors for $\mathrm{AD}$ have been identified. The prevalence of $\mathrm{AD}$ increases with age, diabetes, depression, family history of Parkinson's disease and following head injury or exposure to solvents (Jorm et al., 1991; van Duijn et al., 1991; Ott et al., 1995; Yoshitake et al., 1995; Devanand et al., 1996). Published research further suggests that low education levels are associated with increased prevalence of clinical AD (Gatz et al., 2001; Qiu et al., 2001; Ravaglia et al., 2002). Women also have a higher risk for developing the disease than men, with the risk being markedly increased following menopause (Sherwin, 2002; Sherwin 2003). Additionally, slightly more severe cognitive deficits have been reported in $\mathrm{AD}$ in women compared to men (Buckwalter et al., 1993, Henderson and Buckwalter, 1994). These epidemiological trends may be a consequence of reproductive hormonal changes. Specifically, menopause results in a marked diminution in gonadal estrogen production in women (see Sherwin, 2003, for a review). Estrogen plays a pivotal role in the maintenance and function of neuronal circuits in the brain and in resistance to neuronal damage (McEwen, 2001). The neuroprotective properties of estrogen are thought to be mediated at least in part by anti-amyloidogenic, anti-oxidative and ant-inflammatory mechanisms (reviewed in Barron et al., 2006a). However, limited and somewhat mixed data exist regarding the association between endogenous levels of estrogen and cognitive decline (Manly et al., 2000; Schupf et al., 2003). Based on some of our own findings, we here consider the factors that may be useful in predicting memory decline as a risk factor for Alzheimer's disease in older post-menopausal women.

We have previously noted the relationship between reproductive hormones (estrogen, leutinizing hormone (LH) and follicle stimulating hormone (FSH)) and global cognitive status in a large cohort of over 500 postmenopausal women
(Rodrigues et al., 2008). However, with respect to the symptomatology of early stage $\mathrm{AD}$, specific deficits in episodic learning and memory are the most commonly occurring cognitive signs of the disease (Collie and Maruff, 2000; Remy et al., 2005). We have noted a lack of independent or combined association between endogenous estrogen status and episodic memory capacity in the same cohort of over 500 older postmenopausal women studied by Rodrigues et al. (2008). This finding was observed despite the fact that the relatively large dataset allowed for the application of powerful statistical techniques, specifically with respect to multiple linear regression. This outcome is consistent with our previous report (Rodrigues et al. , 2008) that estrogen did not have a statistically significant impact on global cognitive status in the same cohort; instead, gonadotropins, LH and FSH showed significant associations with global cognition. Further, gonadotropins have been reported to exert a significant role in $\mathrm{AD}$ risk and pathogenesis (reviewed in Barron et al., 2006b). However, in contrast to our findings, other studies have reported that endogenous estrogen does exert a significant effect on cognition (Drake et al., 2000; Senanarong et al., 2002; Wolf and Kirschbaum, 2002; Yaffe et al., 2000), and that estrogen shows no associations with gonadotropin levels (Hoskin et al., 2004; Tsolaki et al., 2005). The discrepancies that exist in these studies merit further consideration, specifically with respect to issues concerning sample size, age of cohort, presence of other $\mathrm{AD}$-associated risk factors (including the APOE $\varepsilon 4$ allele) and the type of cognitive capacity being evaluated.

A specific threshold for estrogen may exist in relation to its influence on cognition: it is possible that clinically measurable effects of estrogen on cognition may only occur at higher concentrations (for example, after exogenous estrogen administration), compared with levels that are naturally present in post-menopausal women. In this context, it is relevant that several studies using measures of episodic memory have found an improvement in cognitive performance after exogenous estrogen replacement therapy (Phillips and Sherwin, 1992; Kampen and Sherwin, 1994; Jacobs et al., 1998; Burkhardt et al., 2004). Indeed, considering the substantial number of investigations into the effects of estrogen administration on memory in post-menopausal women, it is perhaps surprising that relatively little evidence exists concerning the relationship between endogenous estrogen concentration and episodic 
memory. Exogenous treatments of estrogen in postmenopausal women may better match the naturally occurring biological levels found in pre-menopausal women, and may be more likely to have a significant impact on memory and learning. Further, given that the findings the we note here were obtained in a relatively older cohort (aged 75-86 years; Rodrigues et al., 2008), it is possible that women's endogenous estrogen levels in the earlier post-menopause years are more strongly associated with episodic memory functioning.

We further observed in this cohort (Rodrigues et al. , 2008) that the possession of an APOE $\varepsilon 4$ allele did not have a statistically significant impact on verbal episodic memory performance. This finding is again consistent with our previous investigation of global cognitive status in this cohort of older post-menopausal women (Rodrigues et al., 2008). This point notwithstanding, considerable empirical evidence exists regarding the APOE genotype and its role in modifying the risk of age-related cognitive decline and $\mathrm{AD}$. However, the presence of the $\varepsilon 4$ allele is associated with less than $50 \%$ of the population of patients with AD (Evans et al., 1997). Furthermore, this allele may not be a significant risk factor in all ethnic groups (Evans et al., 2003). In this cohort of post-menopausal women, other modulating factors such as education, age and other relevant biomarkers may have attenuated the impact of APOE genotype. We believe that this issue also warrants further discussion and investigation.

Elderly people have an enhanced risk of depressive disorders and symptoms (Wells et al., 1989; Judd and Akiskal, 2000; Judd et al., 2000). Moreover, a number of studies have reported a potentially important link between age-related depression and memory decline (Kral, 1983; Alexopoulos et al., 1993; Devanand et al., 1996). In our cohort (Rodrigues et al., 2008), depression was found not to be a significant factor associated with episodic learning or memory performance. The existing literature suggests that symptoms of depression can have quite specific effects on elements of cognitive functioning. This consideration might be highly relevant in this context.

Our data were consistent with established findings linking increased age, reduced educational level and increased blood-borne beta amyloid to lower episodic memory capacity. Further, one element of memory capacity that was evaluated (delayed recognition performance) was significantly associated with statin use and with hypertension status in this cohort (Rodrigues et al., 2008). Statins are used as pharmaceutical agents to reduce plasma levels of cholesterol, by inhibiting the activity of the enzyme 3-hydroxy-3-methyl-glutaryl- coenzyme. Accumulating evidence suggests that the use of statins lowers the risk of dementia, most likely via amyloid-related mechanisms (Jick et al., 2000; Fassbender et al., 2001; Pedrini et al., 2005). Statins may also improve endothelial homeostasis by increasing the accessibility of nitric oxide (Vaughan, 2003), which may facilitate chemical messaging between cells.

Our findings indicated that hypertension was a risk factor for lower episodic memory capacity. A number of previous studies have linked high arterial blood pressure to increased risk of late-life dementia and cognitive decline (Knopman et al., 2001; van Dijk et al., 2004; Whitmer et al., 2005). Indeed, studies have reported that hypertension may increase the rate of cognitive decline both in patients with $\mathrm{AD}$ (Hanon et al., 2003) and in controls (Bellew et al., 2004). However, other studies have indicated no significant association between hypertension and age-related decline in cognitive performance (Farmer et al., 1987, 1990; Scherr et al., 1991; van Boxtel et al., 1998). With respect to our own findings pertaining to hypertension, high blood pressure may cause cerebrovascular disease (particularly ischemia), including relatively minor but chronic disturbances in cerebral perfusion, producing unfavorable effects on brain cell metabolism (Elias et al., 1993). These changes may increase the likelihood that individuals with incipient AD pathology will express symptoms of dementia, including cognitive decline. Hypertension may also accelerate the AD process directly (Skoog and Gustafson, 2003), insofar as similar biological mechanisms may be involved in the pathogenesis of both hypertension and $\mathrm{AD}$.

In summary, we have noted here that, in our cohort (Rodrigues et al., 2008) age, beta amyloid concentration and hypertension were negatively associated with episodic memory function, whereas education and statin use had positive associations with episodic memory. A relationship between endogenous estrogen and memory functioning was not evident. In future, additional insight will be gained from extended longitudinal investigations of similar cohorts, employing a wider range of cognitive and functional assessments in enriched groups of individuals who are at increased risk of prodromal AD. Further, although a wealth of literature exists on the relationship between reproductive hormones and cognitive decline, $\mathrm{AD}$ risk and $\mathrm{AD}$ pathogenesis, the precise role of estrogen still remains unclear. This is reiterated in the controversy that exists concerning the putative benefits of hormone replacement therapy for improving cognitive capacity and as a therapeutic for $\mathrm{AD}$ (for recent review and meta analysis, see 
Hogervorst et al., 2009). It is clear that in future large clinical studies which control for a number of relevant factors (as adumbrated herein) are required to address some of the discrepancies in the extant literature (Manly et al., 2000; Schupf et al., 2003).

\section{Conflict of interest}

None.

\section{References}

Alexopoulos, G. S., Young, R. C. and Meyers, B. S. (1993). Geriatric depression: age of onset and dementia. Biological Psychiatry, 34, 141-145.

Barron, A. M., Fuller, S. J., Verdile, G. and Martins, R. N. (2006a). Reproductive hormones modulate oxidative stress in Alzheimer's disease. Antioxidants and Redox Signaling, 8, 2047-2059.

Barron, A. M., Verdile, G. and Martins, R. N. (2006b). Gonadotropins: potential targets for preventative and therapeutic interventions in Alzheimer's disease. Future Neurology, 1, 189-202.

Bellew, K. M., Pigeon, J. G., Stang, P. E., Fleischman, W., Gardner, R. M. and Baker, W. W. (2004). Hypertension and the rate of cognitive decline in patients with dementia of the Alzheimer type. Alzheimer's Disease and Associated Disorders, 18, 208-213.

Buckwalter, J. G., Sobel, E., Dunn, M. E., Diz, M. M. and Henderson, V. W. (1993). Gender differences on a brief measure of cognitive functioning in Alzheimer's disease. Archives of Neurology, 50, 757-760.

Burkhardt, M. S. et al. (2004). Estrogen replacement therapy may improve memory functioning in the absence of APOE epsilon4. Fournal of Alzheimer's Disease, 6, 221-228.

Collie, A. and Maruff, P. (2000). The neuropsychology of preclinical Alzheimer's disease and mild cognitive impairment. Neuroscience and Biobehavioral Reviews, 24, 365-374.

Devanand, D. P. et al. (1996). Depressed mood and the incidence of Alzheimer's disease in the elderly living in the community. Archives of General Psychiatry, 53, 175-182.

Drake, E. B. et al. (2000). Associations between circulating sex steroid hormones and cognition in normal elderly women. Neurology, 54, 599-603.

Elias, M. F., Wolf, P. A., D'Agostino, R. B., Cobb, J. and White, L. R. (1993). Untreated blood pressure level is inversely related to cognitive functioning: the Framingham Study. American fournal of Epidemiology, 138, 353-364.

Evans, D. A. et al. (1997). Apolipoprotein E epsilon4 and incidence of Alzheimer disease in a community population of older persons. $\mathcal{F A M A}, 277,822-824$.

Evans, D. A. et al. (2003). Incidence of Alzheimer disease in a biracial urban community: relation to apolipoprotein $\mathrm{E}$ allele status. Archives of Neurology, 60, 185-189.

Farmer, M. E. et al. (1987). Blood pressure and cognitive performance: the Framingham Study. American fournal of Epidemiology, 126, 1103-1114.
Farmer, M. E., Kittner, S. J., Abbott, R. D., Wolz, M. M., Wolf, P. A. and White, L. R. (1990).

Longitudinally measured blood pressure, antihypertensive medication use, and cognitive performance: the Framingham Study. Fournal of Clinical Epidemiology, 43, 475-480.

Fassbender, K. et al. (2001). Simvastatin strongly reduces levels of Alzheimer's disease beta-amyloid peptides Abeta 42 and Abeta 40 in vitro and in vivo. Proceedings of the National Academy of Sciences of the USA, 98, 5856-5861.

Gatz, M., Svedberg, P., Pedersen, N. L., Mortimer, J. A., Berg, S. and Johansson, B. (2001). Education and the risk of Alzheimer's disease: findings from the study of dementia in Swedish twins. Fournals of Gerontology, Series B: Psychological Sciences and Social Sciences, 56, 292-300.

Gilman, S. (1997). Alzheimer's disease. Perspectives in Biology and Medicine, 40, 230-245.

Hanon, O., Seux, M. L., Lenoir, H., Rigaud, A. S. and Forette, F. (2003). Hypertension and dementia. Current Cardiology Reports, 5, 435-440.

Henderson, V. and Buckwalter, J. (1994). Cognitive deficits of men and women with Alzheimer's disease. Neurology, 44, 90-96.

Hogervorst, E., Yaffe, K., Richards, M. and Huppert, F. A. (2009). Hormone replacement therapy to maintain cognitive function in women with dementia. Cochrane Database Systematic Review, 1, CD003799.

Hoskin, E. K., Tang, M. X., Manly, J. J. and Mayeux, R. (2004). Elevated sex-hormone binding globulin in elderly women with Alzheimer's disease. Neurobiology of Aging, 25, 141-147.

Jacobs, D. M. et al. (1998). Cognitive function in nondemented older women who took estrogen after menopause. Neurology, 50, 368-373.

Jick, H., Zornberg, G. L., Jick, S. S., Seshadri, S. and Drachman, D. A. (2000). Statins and the risk of dementia. Lancet, 356, 1627-1631.

Jorm, A. F. et al. (1991). Psychiatric history and related exposures as risk factors for Alzheimer's disease: a collaborative re-analysis of case-control studies. EURODEM Risk Factors Research Group. International Fournal of Epidemiology, 20 (Suppl. 2), S43-S47.

Judd, L. L. and Akiskal, H. S. (2000). Delineating the longitudinal structure of depressive illness: beyond clinical subtypes and duration thresholds. Pharmacopsychiatry, 33, 3-7.

Judd, L. L. et al. (2000). Does incomplete recovery from first lifetime major depressive episode herald a chronic course of illness? American fournal of Psychiatry, 157, 1501-1504.

Kampen, D. L. and Sherwin, B. B. (1994). Estrogen use and verbal memory in healthy postmenopausal women. Obstetrics and Gynecology, 83, 979-983.

Knopman, D. et al. (2001). Cardiovascular risk factors and cognitive decline in middle-aged adults. Neurology, 56, $42-48$.

Kral, V. A. (1983) The relationship between senile dementia (Alzheimer type) and depression. Canadian fournal of Psychiatry, 28, 304-306.

Manly, J. J. et al. (2000). Endogenous estrogen levels and Alzheimer's disease among postmenopausal women. Neurology, 54, 833-837. 
McEwen, B. S. (2001). Plasticity of the hippocampus: adaptation to chronic stress and allostatic load. Annals of the New York Academy of Sciences, 933, 265-277.

Ott, A. et al. (1995). Prevalence of Alzheimer's disease and vascular dementia: association with education. The Rotterdam Study. BMF, 310, 970-973.

Pedrini, S., Carter, T. L., Prendergast, G., Petanceska, S., Ehrlich, M. E. and Gandy, S. (2005). Modulation of statin-activated shedding of Alzheimer APP ectodomain by ROCK. PLOS Medicine, 2, 69-78.

Phillips, S. M. and Sherwin, B. B. (1992). Effects of estrogen on memory function in surgically menopausal women. Psychoneuroendocrinology, 17, 485-495.

Qiu, C., Backman, L., Winblad, B., Aguero-Torres, H. and Fratiglioni, L. (2001). The influence of education on clinically diagnosed dementia: incidence and mortality data from the Kungsholmen Project. Archives of Neurology, 58, 2034-2039.

Ravaglia, G. et al. (2002). Education, occupation, and prevalence of dementia: findings from the Conselice study. Dementia and Geriatric Cognitive Disorders, 14, 90100.

Remy, F., Mirrashed, F., Campbell, B. and Richter, W. (2005). Verbal episodic memory impairment in Alzheimer's disease: a combined structural and functional MRI study. Neuroimage, 25, 253-266.

Rodrigues, M. A. et al. (2008). Gonadotropins and cognition in older women. Fournal of Alzheimer's Disease, 13, 267-274.

Scherr, P. A., Herbert, L. E., Smith, L. A. and Evans, D. A. (1991). Relation of blood pressure to cognitive function in the elderly. American fournal of Epidemiology, 134, 1303-1315.

Schupf, N. et al. (2003). Onset of dementia is associated with age at menopause in women with Down's syndrome. Annals of Neurology, 54, 433-438.

Senanarong, V. et al. (2002). Endogenous estradiol in elderly individuals: cognitive and noncognitive associations. Archives of Neurology, 59, 385-389.

Sherwin, B. B. (2002). Randomized clinical trials of combined estrogen-androgen preparations: effects on sexual functioning. Fertility and Sterility, 77 (Suppl. 4), S49-S54.

Sherwin, B. B. (2003). Estrogen and cognitive functioning in women. Endocrine Reviews, 24, 133-151.

Skoog, I. and Gustafson, D. (2003). Hypertension, hypertension-clustering factors and Alzheimer's disease. Neurological Research, 25, 675-680.

Tsolaki, M. et al. (2005). Serum estradiol, progesterone, testosterone, FSH and $\mathrm{LH}$ levels in postmenopausal women with Alzheimer's dementia. Hellenic fournal of Nuclear Medicine, 8, 39-42.

van Boxtel, M. P., Buntinx, F., Houx, P. J., Metsemakers, J. F., Knotterus, A. and Jolles, J. (1998). The relation between morbidity and cognitive performance in a normal aging population. fournals of Gerontology, Series A: Biological Sciences and Medical Sciences, 53, M147-M154.

van Dijk, E. J. et al. (2004). The association between blood pressure, hypertension, and cerebral white matter lesions: cardiovascular determinants of dementia study.

Hypertension, 44, 625-630. van Duijn, C. M., Stijnen, T. and Hofman, A. (1991). Risk factors for Alzheimer's disease: overview of the EURODEM collaborative re-analysis of case-control studies. EURODEM Risk Factors Research Group. International Fournal of Epidemiology, 20 (Suppl. 2), S4-S12.

Vaughan, C. J. (2003). Prevention of stroke and dementia with statins: effects beyond lipid lowering. American fournal of Cardiology, 91, 23B-29B.

Wells, K. B. et al. (1989). The functioning and well-being of depressed patients: results from the Medical Outcomes Study. $\mathcal{F} A M A, 262,914-919$.

Whitmer, R. A., Sidney, S., Selby, J., Johnston, S. C. and Yaffe, K. (2005). Midlife cardiovascular risk factors and risk of dementia in late life. Neurology, 64, 277-281.

Wolf, O. T. and Kirschbaum, C. (2002). Endogenous estradiol and testosterone levels are associated with cognitive performance in older women and men. Hormones and Behavior, 41, 259-266.

Yaffe, K., Lui, L.-Y., Grady, D., Cauley, J., Kramer, J. and Cummings, S. R. (2000). Cognitive decline in women in relation to non-protein-bound estradiol concentrations. Lancet, 356, 708-712.

Yoshitake, T. et al. (1995). Incidence and risk factors of vascular dementia and Alzheimer's disease in a defined elderly Japanese population: the Hisayama Study. Neurology, 45, 1161-1168.

MARK A. Rodrigues, ${ }^{1}$ Jonathan K. Foster, ${ }^{1,6}$ GiUseppe Verdile, ${ }^{1}$ Karen Joesbury, ${ }^{1}$ Richard Prince, ${ }^{2}$ Amanda Devine, ${ }^{3}$ Pankaj Mehta, ${ }^{4}$ JOHN BEILBY ${ }^{5}$ AND RALPH N. MARTINS ${ }^{1}$

${ }^{1}$ Sir James McCusker Alzheimer's Disease Research Unit, Hollywood Private Hospital; Centre of Excellence in Alzheimer's Disease Research and Care, School of Exercise, Biomedical and Health Sciences, Edith Cowan University, Joondalup, Western Australia; and School of Psychiatry and Clinical Neurosciences, University of Western Australia, Australia

${ }^{2}$ School of Medicine and Pharmacology, Sir Charles Gairdner Hospital, University of Western Australia and Department of Endocrinology and Diabetes, Sir Charles Gairdner Hospital, Western Australian Institute for Medical Research, Western Australia, Australia

${ }^{3}$ School of Exercise, Biomedical and Health Sciences, Edith Cowan University, Joondalup, Western Australia, Australia

${ }^{4}$ Institute for Basic Research in Developmental Disabilities, New York, U.S.A.

${ }^{5}$ PathCentre, Western Australian Centre for Pathology and Medical Research, Clinical Biochemistry, Nedlands, Western Australia, Australia

${ }^{6}$ Neurosciences Unit, Health Department of WA, Perth, Western Australia, Australia Email: j.foster@ecu.edu.au 